EPiC Series in Computing
Volume 58, 2019, Pages 438-443
Proceedings of 34th International Confer-
ence on Computers and Their Applications

\title{
Improving the Performance of 3D Printed Molds for Plastic Injection Molding
}

\author{
Faryar Etesami, Christopher D. Mullens, Ramsey G. Sahli, and Trey J. Webb \\ Mechanical and Materials Engineering Department, Portland State University \\ Portland, Oregon, 97201, USA \\ etesamf@pdx.edu
}

\begin{abstract}
Additive manufacturing technology has become a viable solution for making molds for plastic injection molding applications. The molds are usually made of high temperature plastic resins suitable for plastic injection molding. Molding resins have superior mechanical properties necessary to withstand the high temperatures and pressures of the injection molding process. It is known that high temperature mechanical properties of resins influence mold performance but it is not established which properties are most important and to what extent they influence the mold performance. Identifying the most important properties influencing mold performance would help resin manufacturers to develop better mold-making materials. In order to study the performance of mold materials we have built a device for measuring the mechanical properties of 3D printed resins including their strength, surface hardness, and wear resistance at molding temperatures of up to $260^{\circ} \mathrm{C}$. We then quantified the mechanical properties of three high-temperature resins along with ABS at the injection molding temperatures. This paper describes the test device and the results of characterizing the mechanical properties of the selected plastics.
\end{abstract}

\section{Introduction}

Rapid tooling (RT) refers to creating molds or dies for injection molding, casting, or forming processes using additive manufacturing technology (AM). It is believed that in many cases additively manufactured tools can substantially reduce production costs and lead-times compared to the traditional tool-making processes [1] (journal). AM technology can be used to create durable metal molds or plastic molds. Plastic molds, however, can be made much quicker and at a substantially lower cost than metal molds. The focus of this research is on low-volume production of small parts where the number of parts range from a handful to a few hundred. For low-volume applications, or 
functional prototyping, where the tolerances are not tight, the use of traditional metal molds or even AM metal molds lead to excessive costs and lead times that can be avoided using polymeric molds [2] (journal) and [3] (book). A limitation of plastic molds is their low heat transfer capability resulting in slower production rates compared to metal molds. This limitation can, to a certain degree, be alleviated using conformal cooling channels [4] (journal) and [5] (journal). In selecting the best mold materials, an important research question is how to identify the most important properties necessary to predict the performance of 3D printed mold materials. Currently, the suitability of mold materials is mainly judged by their heat deflection temperature (HDT). The HDT, which is defined in detail by ASTM D648 Standard [6] (standard), is the temperature at which a polymer or plastic sample deforms a specific amount under a specific load in a 3-point bending test. For amorphous polymers the HDT closely follows the glass transition temperature. It is believed that HDT is one of the properties that correlates well with the quality and integrity of molds used in injection molding. It is well-known that the mechanical properties of 3D printed materials can be very different than cast materials due to directional properties, partial solidity, and print technology [7] (journal). Our research test device measures the material deflection in a manner similar to the standard HDT devices. The device creates an HDT profile that includes a range of temperatures. An HDT profile is more useful for research purposes than a single HDT value. The device also measures the material surface hardness at high temperatures. The resulting performance curve characterizes the surface hardness change as a function of temperature. We suspect that surface hardness is also a good indicator for evaluating the mold resistance to surface damage. The third type of test measures the material's wear characteristics at elevated temperature. Plastic molds that survive cracking or chipping eventually fail due to wear, which results in unacceptable part tolerances and rounding of sharp edges.

\section{The Material Test Device (MTD)}

Figure-1 shows the CAD model of the MTD. Figure-2 shows the actual device.

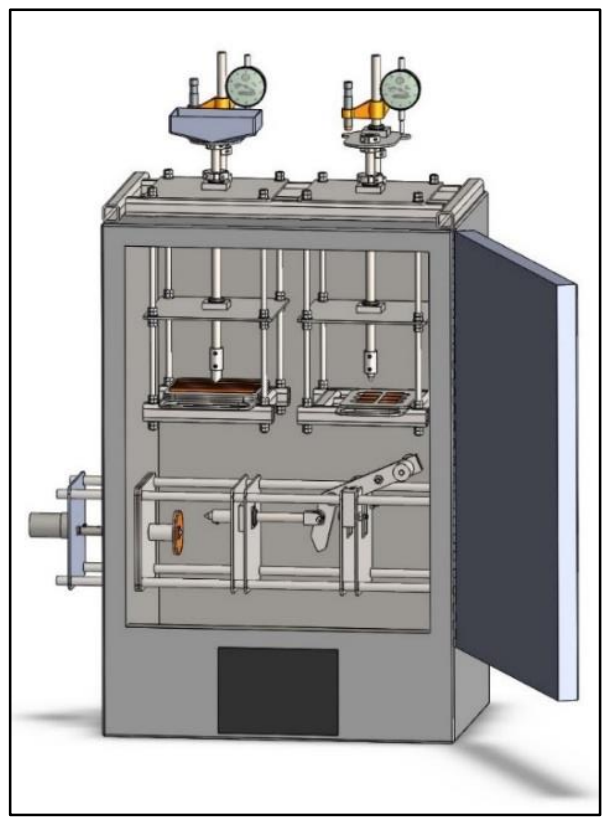

Figure-1. The CAD model of MTD

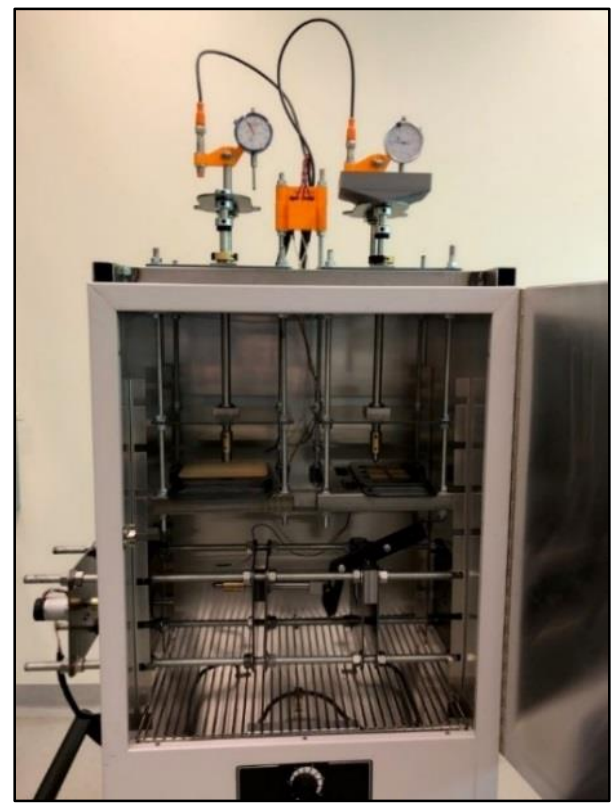

Figure-2. The actual material test device 
The box is a temperature-controlled oven capable of creating and maintaining temperatures up to 260 degrees $\mathrm{C}$. This temperature limit is suitable for injection molding of common plastics such as polypropylene, nylon, ABS, and HDPE. The top row trays are for three-point bending test and surface hardness test while the lower portion of the box houses the wear test. The device is also capable of measuring the creep characteristics of various polymers.

The deflections for the three-point bending device and displacements for the hardness are measured by high accuracy inductive displacement sensors. Dial indicators are also used for instant visual feedback and for verification of inductive sensor responses. Thermocouples are placed near the test samples. The standard test requires the samples to be placed in an oil bath for better heat transfer such that the temperature of the oil and the sample are close [5] (standard). This is necessary to eliminate variations among standard machines. Our device does not use an oil bath as we use the MTD for comparative testing. As long as the same test machine is used, there is no need to have high precision alignments for loading, oil bath with stirring fans, or a precise temperature profile of 2 degrees $\mathrm{C}$ per minute. Not having the complexity of conforming to a standard test makes the MTD much easier to use and maintain at a fraction of the cost of a certified standard device.

The test sample geometry is the same as the standard samples. The loading is primarily provided by the weight of the rod and associated parts. The samples are 3D printed and when loaded by the bar alone (and some small weight adjustments) create an outer fiber stress of $1.82 \mathrm{Mpa}$ or $264 \mathrm{psi}$, which is compatible with the standard test. The sample width and thickness are measured and the necessary load is calculated based on the bending formula as prescribed by the Standard. The hardness tester uses the same loading mechanism. Inductive sensors measure the depth of penetration as a function of temperature. The resulting profile is similar to HDT except that the deflection is due to surface hardness resistance, rather than bending resistance. Either the 3-point bending test set up or the harness test set up can be used to measure the creep parameters of the test polymers as well. Having the creep parameters allows us to perform strength and deflection simulations when the mold is injected by hot high pressure plastic.

The wear test mechanism of MTD is composed of a motor that rotates a circular specimen and a linkage that applies a load to wear tip that slides on the surface of the specimen. The system is designed such that the worn away material falls down and would not get embedded into the wear tip. Figure-3 shows the section-cut CAD model of mechanism for surface wear measurement indicating the contact mechanism, the wear tip, and the specimen. The load linkage can be better seen in Figure-1.

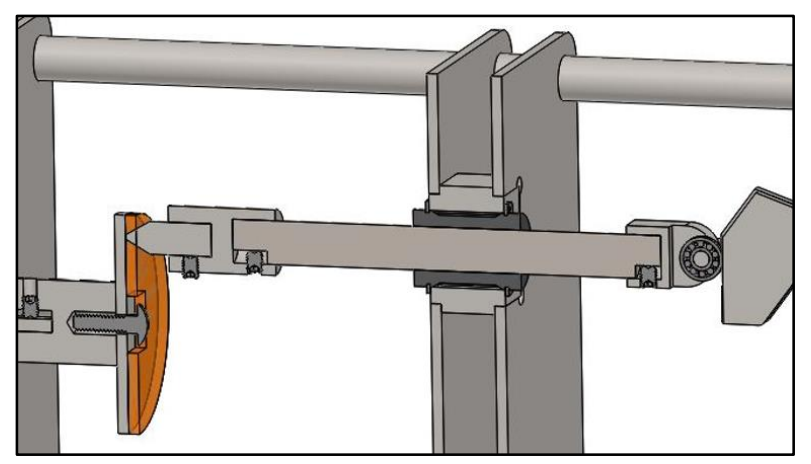

Figure-3. The wear test mechanism. 
Figure-4 shows the geometry of the tips for bending test. The test tray is designed for simultaneous testing of multiple specimens by allowing to quickly switch between different materials as a target temperature is reached. A similar tray design allows simultaneous tests for hardness measurement as well. We are currently experimenting with different wear tips to determine the best geometry and materials that closely simulates the flow of hot plastic on a surface.

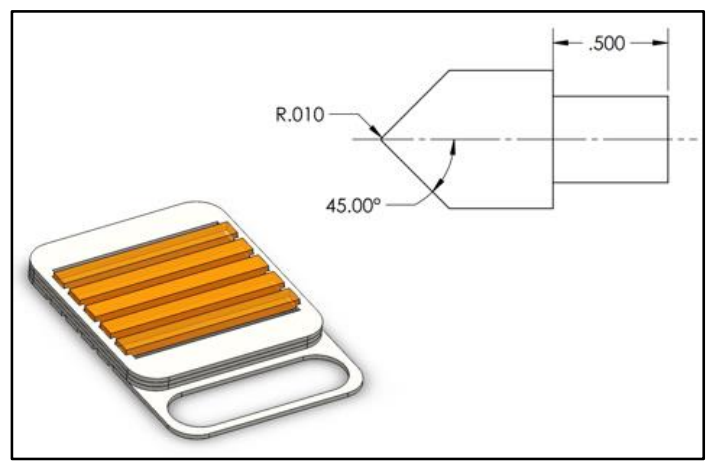

Figure-4. The bending test tray and tip geometry.

\section{Test Procedure}

The materials and 3D printing processes used to create the mechanical properties curves are:

- ABS filament made with an FDM machine with solid fill pattern.

- High temperature resin from Formlabs [8] (online) made with an SLA process.

- Onyx filament from Markforged [9] (online) made on an FDM machine.

- High strength high temperature (HSHT) fiberglass-reinforced composite filament [10] (online) from Markforged made on an FDM machine

One sample of each material was used for 3-point bending and hardness wear tests. The results of the wear tests or creep tests are not reported in this paper. For 3-point bending tests the samples were measured and loads were calculated to create a stress of $1.82 \mathrm{Mpa}$ in the outer fibers consistent with ASTM-D648 standard. The load was then applied at room temperature for 5 minutes to allow the sample go through room-temperature creep. Then the oven was turned on until the deflections exceeded the heat deflection value of $0.25 \mathrm{~mm}$. The oven was modified to create a temperature profile of about 2 degrees $\mathrm{C}$ per minute consistent with the requirements of the Standard test. Therefore the results of the 3-point bending test should be in agreement with the HDT of certified testing machines in an edgeloading set up. Since this device is used for comparative experiments, there is no need to report certified values of HDT. However, the results should be in general agreement with values reported with certified HDT machines. The hardness test is similarly performed with exactly the same load every time. The wear test, which is not reported in this paper, is performed at the highest temperature the oven can generate. Each sample undergoes a specific number of rotations at a fixed load. The amount of wear is measured as the difference in weight of the sample before and after the test, or as the depth of the groove made by the wear test tip. 


\section{Characteristic Curves}

Figure-5 shows the relationship between the test specimen deflection as a function of temperature and time. Since the time-temperature profile is nearly identical for all tests, the resulting graph is a comparable indictor of deflection due to both time and temperature.

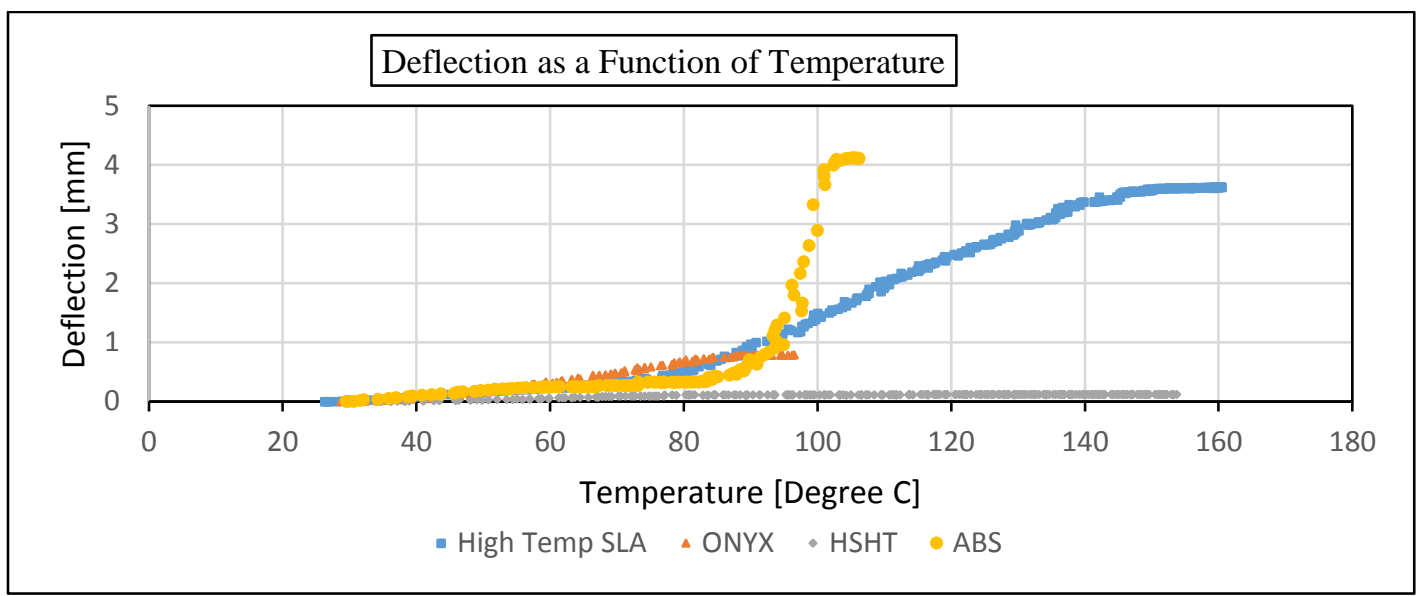

Figure-5. Heat Deflection Curves

This plot clearly shows that high temperature stiffness of Markforged HSHT filament with the FDM process exhibits the lowest deflection of the materials tested. In particular, Formlabs high temperature resin shows much more deflection than the Markforged HSHT material. In addition, although the performance of ABS rapidly deteriorates at higher temperatures, it may be suitable for molding low temperature plastics. Figure- 6 shows the relationship between the test specimen depth of indentation as a function of temperature in the hardness test. The ABS response in truncated as the indentation for ABS increases at the same slope until the back end of the specimen is punctured. Again, the fiberglassfilled HSHT filament of Markforged shows the highest value of surface hardness.

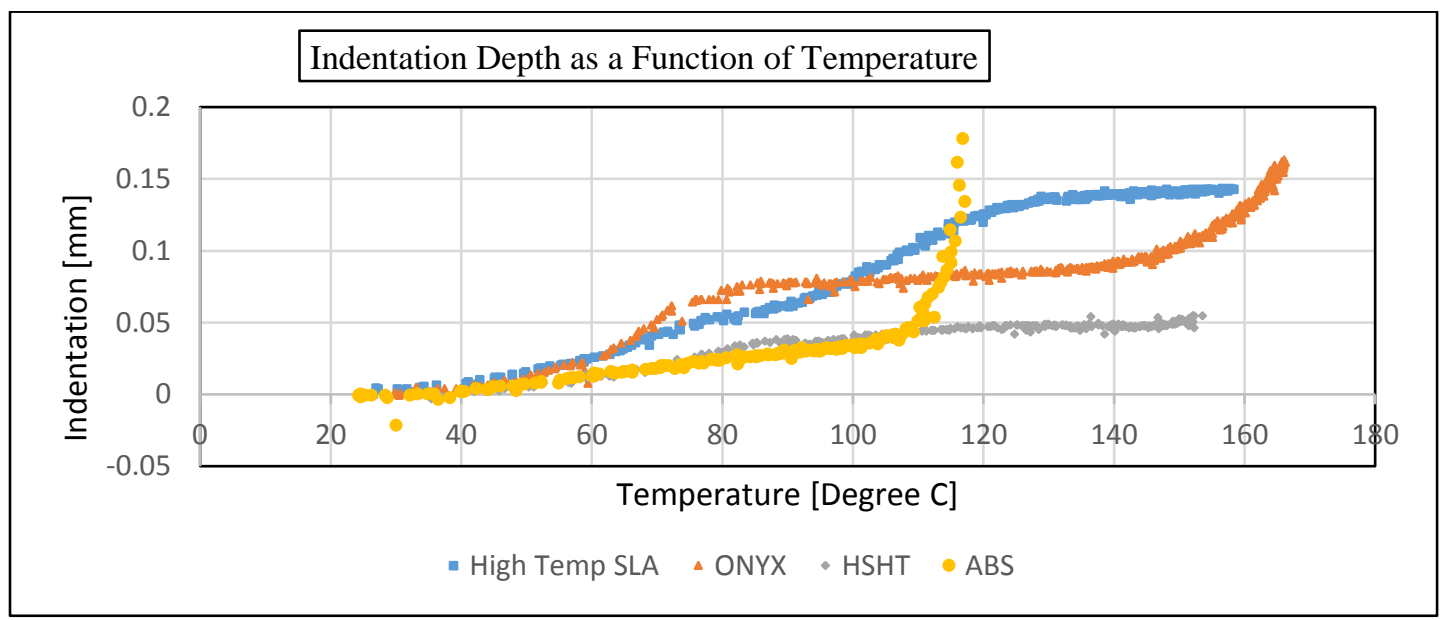

Figure-6. Heat Indentation Curve 


\section{Conclusions and Future Work}

Performance of 3D printed plastic molds is influenced by the mechanical and thermal properties of the mold material at high temperatures as well the 3D printing process. Since molds fail in a variety of ways, it is important to establish the properties responsible for each mode of failure. Our MTD device can be used to accurately characterize the mechanical properties of 3D printed specimens quickly and inexpensively. We compared the bending deflections and surface hardness of four candidate materials. The results show a significant difference in how these materials respond to load, temperature, and time. The overall objective is to relate these mechanical properties to the performance of molds created by these materials and processes. The results of this research is likely to help 3D printing manufacturers develop better materials for mold-making. In addition, the design guidelines developed through this research will help designers match their requirements for plastic part materials and geometry to the most suitable 3D printing material and technology. There are many other candidate materials available in the market that are believed to be suitable for 3D printed molds. The future work in this research includes testing other leading materials such as 3D Systems VisiJetSL HiTemp [11] (online), and a number of materials from Stratasys [12] (online). The next phase of this research proceeds with making molds from the best candidate materials to evaluate their performance in injection molding of various plastics and to develop predictive models for how mold performance relate to the mechanical properties data obtained by MTD.

\section{References}

[1] Azhar Equbal, Anoop Kumar Sood, Mohammad Shamim. Rapid Tooling: A Major Shift in Tooling Practice. Journal of Manufacturing and Industrial Engineering, Vol. 14, No. 3-4, 2015

[2] Lidsey Frick. 3D Print Plastic Molds and Tooling, Machine Design, Nov. 2013. Pages 74-75

[3] Ian Gibson, David W. Rosen, Brent Stucker. Additive Manufacturing Technologies. SpringerVerlag 2010

[4] Chil-Chyuan Kuo, Zheng-Yan You. A Cost-effective Approach for Rapid Fabricating Cooling Channels with Smooth Surface. The International Journal of Advanced Manufacturing Technology, Vol 95, Issues 1-4, Feb 2018. Pages 1003-1013.

[5] Chil-Chyuan Kuo, Zheng-Yan You. Development of Injection Molding Tooling with Conformal Cooling Channels Fabricate by Optimal Process Parameters. The International Journal of Advanced Manufacturing Technology, Vol 96, Issues 1-4, Nov 2017. Pages 1135-1141.

[6] ASTM D648-18, Standard Test Method for Deflection Temperature of Plastics Under Flexural Load in the Edgewise Position, ASTM International, West Conshohoken, PA, 2018, www.astm.org.

[7] John Ryan Dizon, Alejandro Espera jr, Qiyi Chen, Rigoberto Advincula. Mechanical Characterization of 3D-printed Polymers. Additive Manufacturing, Vol 20, Mar. 2018, PP 44-67.

[8] Formlabs. High Temperature Resin https://formlabs.com/store/us/form-2/materials/high-temp$\underline{\text { resin/ }}$

[9] Markforged. Fiber glass filled high strength high temperature filament (HSHT). https://markforged.com/blog/announcing-high-temperature-materials-hsht-fiberglass/

[10] Markforged. Onyx filament. https://markforged.com/product/onyx-filament/

[11] 3D System Inc. https://www.3dsystems.com/materials/visijet-sl-hitemp

[12] Stratasys Inc. https://www.stratasys.com/ 